\title{
Produção de forragem e distribuição espacial de árvores de pequi em sistema silvipastoril
}

\author{
Henrique Guimarães de FAVARE ${ }^{1 *}$, Antonio de Arruda TSUKAMOTO FILHO ${ }^{2}$, \\ Joadil Gonçalvez de $\mathrm{ABREU}^{3}$, Lilian Guimarães de FAVARE², Reginaldo \\ Brito da COSTA ${ }^{2}$, Maria Corette PASA $^{2}$ e Laércio COUTO ${ }^{4}$
}

\author{
${ }^{1}$ Programa de Pós-Graduação em Agricultura Tropical, Universidade Federal de Mato Grosso, Cuiabá, MT, Brasil. \\ ${ }^{2}$ Programa de Pós-Graduação em Ciências Florestais e Ambientais, Universidade Federal de Mato Grosso, Cuiabá, MT, Brasil. \\ ${ }^{3}$ Faculdade de Agronomia e Zootecnia, Universidade Federal de Mato Grosso, Cuiabá, MT, Brasil. \\ ${ }^{4}$ Departamento de Engenharia Florestal, Universidade Federal de Viçosa, Viçosa, MG, Brasil \\ ${ }^{*}$ E-mail: guimaraesfavare@gmail.com
}

Recebido em fevereiro/2018; Aceito em maio/2018.

\begin{abstract}
RESUMO: Objetivou-se determinar a distribuição espacial de árvores nativas de pequi e a produção de pasto na estação seca e chuvosa. $\mathrm{Na}$ área de estudo realizou-se o inventário e mapeamento de indivíduos de pequi e sua distribuição espacial foi verificada pelo teste $\mathrm{k}$ de Ripley sobre a população total e em função de classes de área de copa. Para a análise do rendimento da matéria seca, foram coletadas amostras de forragem sob a copa das árvores e nas áreas de pastagem a pleno sol, separadas em lâmina de folha, bainha + colmo e material senescente, no final das estações chuvosa e seca. Os dados foram submetidos ao teste $\mathrm{F}$ e teste $\mathrm{t}$, ambos com significância de $10 \%$. Foram observadas 139 árvores remanescentes de pequi com área de copa média de $148 \mathrm{~m}^{2}$, distribuídas de modo agregado, com tendência aleatoriedade nas classes de menor e maior área de copa. Na condição sombreada houve incremento de forragem independente das estações estudadas. O sombreamento favorece a produção do pasto nos períodos de seca e chuva, com redução do material senescente.
\end{abstract}

Palavras-chave: Caryocar brasiliensis Camb., teste $k$ de Ripley, pasto sombreado, integração.

\section{Forage production and spatial distribution of pequi trees in the silvipastoril system}

\begin{abstract}
The objective was to determine the spatial distribution of pequi native trees and pasture production in dry and rainy seasons. In the study area, the inventory and mapping of pequi individuals was performed and their spatial distribution was verified by the Ripley $\mathrm{k}$ test on the total population and as a function of crown area classes For the analysis of the dry matter yield, forage samples were collected under the canopy of the trees and in the pasture areas in full sun, separated in leaf blade, sheath + stem and senescent material, at the end of the rainy and dry seasons. Data were submitted to the $\mathrm{F}$ test and t test, both with a significance of $10 \%$. A total of 139 remaining pequi trees with a median canopy area of $148 \mathrm{~m}^{2}$, distributed in an aggregate manner, were observed, with a tendency to randomness in the classes of smaller and larger crown area. In the shaded condition there was increment of forage independent of the studied stations. Shading favors pasture production during periods of drought and rain, with reduction of senescent material.
\end{abstract}

Keywords: Caryocar brasiliensis Camb., Ripley's k test, shaded grass, integration.

\section{INTRODUÇÃO}

O desmatamento das áreas de cerrado é preocupante, mas por outro lado algumas estratégias são implementadas para tentar minimizar esse problema, com possibilidade de aproveitamento de espécies arbóreas nativas em diferentes tipos de sistemas de uso do solo. Essas espécies nativas com características potenciais podem ser mantidas no seu local de origem para proporcionar alternativas econômicas associadas à conservação do ecossistema, conforme observado no estudo de Meira et al. (2016). É expressivo o número de espécies arbóreas nativas com potencial de ser utilizado na produção comercial e nos sistemas integrados, mas ainda são poucos os estudos com essas espécies, como o que foi realizado por Calil et al. (2016).

O pequi (Caryocar brasiliense Camb.), espécie marcante da flora brasileira, tem destaque no cerrado devido sua extensa distribuição pelo bioma e por sua importância social, cultural e econômica de alcance internacional (AFONSO; ANGELO,
2009). Seu corte é proibido por lei no Brasil (IBAMA, 2018) e no Estado de Minas Gerais (ALMG, 2018). Associar atividades agroflorestais em áreas nativas remanescentes com esta espécie agrega renda à propriedade rural, devido à exploração de mais produtos comercializáveis e a minimização da degradação do ambiente com o aumento sustentável da produção agropecuária (KICHEL et al., 2014).

Os sistemas integrados, de maneira geral, são complexos, visto que o sinergismo entre os componentes envolvidos é base fundamental para consolidação da prática. Pouco se sabe sobre a distribuição das árvores remanescentes nas pastagens, principalmente para as nativas do cerrado, e como as decisões de manejo por parte dos produtores refletem sobre as próprias árvores, impactam na produtividade agrícola e no ambiente (HARVEY et al., 2011). No entanto, observam-se melhorias diretas na produtividade e na qualidade do pasto em condição de sombreamento, além da maior perspectiva de sucesso na implantação, devido adaptabilidade da nativa à condição 
regional, podendo assim serem preservadas para a formação do pasto (ABREU et al., 2016).

Para o produtor, ter árvores pré-estabelecidas na área, é de interesse financeiro visto a onerosidade, o tempo gasto para plantio, e o desenvolvimento do componente florestal. Ao considerar o uso de espécies nativas como componente arbóreo do sistema silvipastoril, alguns aspectos característicos da estrutura e biodiversidade se assemelham às condições naturais, com tendência a uma maior sustentabilidade da produção e equilíbrio do ambiente. Deste modo objetivou-se determinar o padrão de distribuição espacial de árvores remanescentes de pequi e a produção de pasto na época seca e chuvosa.

\section{MATERIAL E MÉTODOS}

O estudo foi realizado em propriedade rural do município de Cáceres-MT, nas coordenadas geográficas $15^{\circ} 58^{\prime} 25,49^{\prime \prime} \mathrm{S}$ e 57³4'36,11" W. Segundo classificação de Köppen, a região apresenta clima tropical quente semiúmido (Aw), com duas estações bem definidas, inverno seco e chuvas no verão, temperatura máxima anual $31,5^{\circ} \mathrm{C}$ e mínima $20,1^{\circ} \mathrm{C}$. O solo do local é classificado como Latossolo Vermelho Amarelo, de textura franco arenosa (EMBRAPA, 2006).

A área do estudo possui o total de 40 ha, e tornou-se sistema silvipastoril a partir da inserção de forrageiras associadas com as árvores remanescentes de pequi, além de outras espécies nativas do cerrado, as quais não foram utilizadas para o estudo. $\mathrm{O}$ pasto misto foi formado com sementes de gramíneas e uma leguminosa, sendo elas: Panicum maximum Jacq. cv. Mombaça; Brachiaria brizantha Hochst Stapf cv. Xaraés; Panicum sp. cv Massai; Brachiaria brizantha Hochst Stapf cv. Marandu; Estilosantes Campo Grande (Stylosanthes capitata Vog. x Stylosanthes macrocephala M. B. Ferr e S. Costa), e a semeadura feita a lanço com Distribuidor Vicon. Depois de formado, o pasto foi utilizado para alimentação de bovinos por um período de dois meses e, posteriormente, vedado por três meses para descanso.

\subsection{Inventário das árvores de pequi e posição geográfica}

Foi realizado o inventário de prospecção (censo) da população de pequi presente na área de estudo e os indivíduos identificados por meio de placas. Consideraram-se adultos todos aqueles que possuíam $5 \mathrm{~cm}$ ou mais de diâmetro de fuste a $0,30 \mathrm{~m}$ do solo. As variáveis mensuradas foram: circunferência altura do solo (CAS) e posteriormente cálculo de diâmetro à altura do solo (DAS), altura total (Ht), altura sob copa, bem como quatro raios de projeção de copa nos sentidos norte, sul, leste e oeste. As alturas foram medidas com hipsômetro de Blume-Leiss e as circunferências com fita métrica. Para o cálculo da área de copa utilizou-se fita métrica, tomando como ponto de origem o centro do tronco distanciando-se até o limite da copa, considerando-se a direção orientada pelos pontos cardeais (SCOLFORO; MELLO, 2006).

A distribuição dos indivíduos e o mapeamento da área foi efetuado usando o sistema de coordenadas retangulares (UTM), através de georreferenciamento com GPS de precisão, e posteriormente exportado para o TrackMaker 3.5 (Figura 1).

A distribuição espacial do pequi foi verificada analisando a população e a área de copa das árvores (AC). A seleção das classes de área de copa para a análise foi feita a partir do desvio padrão (S) da área de copa das árvores de pequi, conforme descrito por Valério et al. (2009). Através do $\mathrm{S}$ foram estabelecidas cinco classes amostrais, sendo uma classe representando à média e as outras quatro a média mais e menos um e dois $\mathrm{S}$.

As classes de área de copa (AC) representaram os seguintes intervalos populacionais: Classe $1\left(0,00 \mathrm{~m}^{2}\right)$ : intervalo da menor $\mathrm{AC}$ da população até $\mathrm{AC}$ de $30,8 \mathrm{~m}^{2}$; Classe $2\left(70,10 \mathrm{~m}^{2}\right)$ : intervalo da AC 30,9 até 109,4 $\mathrm{m}^{2}$; Classe $3\left(148,70 \mathrm{~m}^{2}\right)$ : intervalo da AC 109,5 até $188,0 \mathrm{~m}^{2}$; Classe 4 $\left(227,30 \mathrm{~m}^{2}\right)$ : intervalo da AC 188,1 até 266,6 $\mathrm{m}^{2}$; Classe 5 $\left(306,00 \mathrm{~m}^{2}\right)$ : intervalo da AC 266,7 $\mathrm{m}^{2}$ até a maior AC da população.

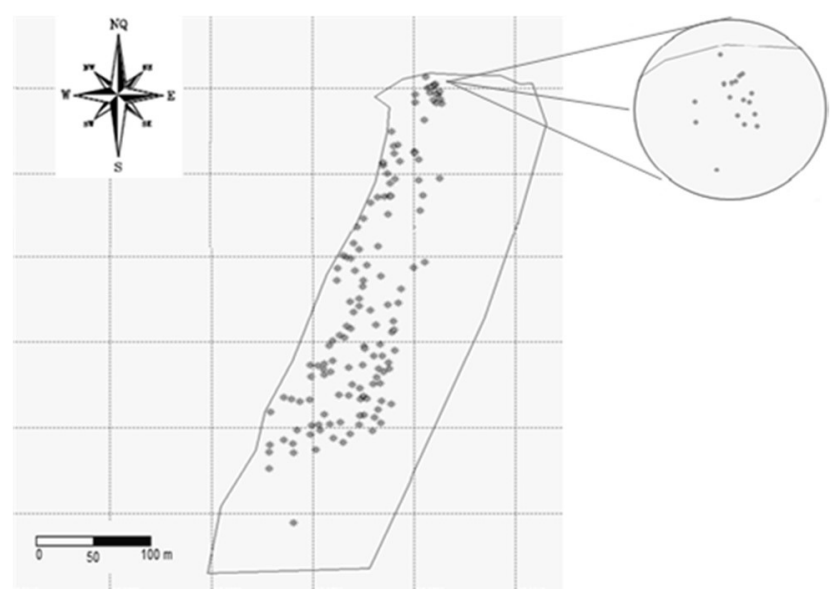

Figura 1. Distribuição das árvores de pequi com diâmetro do fuste $<$ $5 \mathrm{~cm}$ a $0,30 \mathrm{~m}$ do solo.

Figure 1. Distribution of pequi trees with stem diameter $<5 \mathrm{~cm}$ to 0.30 $\mathrm{m}$ above the ground.

Para averiguação da homogeneidade na densidade de distribuição da população de pequi, as coordenadas coletadas foram analisadas pelo estimador de densidade de Kernel (CÂMARA; CARVALHO, 2004). A análise da distribuição espacial foi realizada a partir da Função K de Ripley (RIPLEY, 1981), com o intuito de detectar o padrão espacial em diferentes escalas simultaneamente e testar a independência espacial entre grupos de árvores (BATISTA; MAGUIRE, 1998).

Assim, se os envelopes observados estiverem dentro da confiança dos envelopes (oscila em torno de zero da transformada $L(r)$ ), pode-se dizer que o padrão espacial é aleatório, e os valores fora dos envelopes, a hipótese nula é rejeitada. Desta forma, os valores positivos são considerados como padrão do tipo agregado e se negativos o seu padrão é considerado regular (ASSUNÇÃO; LOPES, 2015). Esta função calcula a média geral dos números dos pontos que caem dentro da área circular de busca do raio $s$. A estimativa é dada por:

$\hat{K}(s)=\frac{1}{\hat{\lambda} n} \sum_{i=1}^{n} \sum_{\substack{j=1 \\ i \neq j}}^{n} w_{I}^{-1}\left(x_{i}, x_{j}\right) I\left(|| x_{i}-x_{j}||<s\right)$

(Equação 1)

Em que: $n$ é número de árvores na região de estudo; $\| \mathrm{X}_{\mathrm{i}}-$ $\mathrm{X}_{\mathrm{j}} \|$ é distância euclidiana entre as localizações $\mathrm{X}_{\mathrm{i}}$ e $\mathrm{X}_{\mathrm{j}}$; $s$ é um vetor arbitrário de distância; $\mathrm{W}_{\mathrm{i}} \mathrm{I}\left(\mathrm{X}_{\mathrm{i}}, \mathrm{X}_{\mathrm{j}}\right)$ é a função de correção para efeito de borda, a qual representa a proporção de circunferência com centro em $\mathrm{X}_{\mathrm{j}}$ e com raio $\left\|\mathrm{X}_{\mathrm{i}}-\mathrm{X}_{\mathrm{j}}\right\|$ que está fora da região de estudo; $\hat{\lambda}=\mathrm{n} /|\mathrm{A}|$ é o número de árvores 
dividido pela área da região de estudo, sendo um estimador não viciado de intensidade do processo; I (U) é uma função indicadora que assume valor 1 sempre que a condição $U$ for verdadeira, e o valor 0 quando for falsa.

Em regiões de estudo com formato regulares, o estimador de Ripley será não viciado para distâncias inferiores à metade do menor lado do retângulo (DIGGLE, 2003). Para facilitar a visualização dos resultados, a função $\mathrm{K}$ é transformada para $L(s)$, em que:

$$
\widehat{L(s)}=\sqrt{\widehat{K(s)} / \pi}-s
$$

Se o padrão for completamente aleatório, a função $L(s)$ se apresenta como uma linha horizontal sobre o eixo das abcissas. A agregação em padrão espacial se refletirá em número maior de árvores que o esperado pela completa aleatoriedade espacial, e a função assume valores positivos. Se o padrão apresentar regularidade na localização das árvores, o número destas será menor que o esperado pela Completa Aleatoriedade Espacial (CAE) e a função $L(s)$ assume valores negativos. Assim as hipóteses podem ser construídas da seguinte forma: $\mathrm{H}_{0}$ : Poisson $L(s)=0$; e $\mathrm{H}_{1}: L(s) \neq 0$, em que: $L(s)>0$, o padrão é agregado; $L(s)<0$, o padrão é uniforme.

Foram testados os desvios em relação à CAE, para a função univariada (BATISTA; MAGUIRE, 1998). Para este estudo, a CAE foi examinada mediante envelopes de confiança construídos através 1000 simulações Montecarlo, produzindo envelopes de confiança de 99,9\%.

Os cálculos foram realizados no programa estatístico R, com o pacote Spatstat (BADDELEY, 2010). A avaliação da relação existente entre os pares de indivíduos foi calculada automaticamente pelo programa computacional. Em regiões que se deseja estudar onde a área tem formato geométrico, como o caso deste estudo, o estimador da função K de Ripley é gerado em uma distância que pode oscilar até metade do menor lado da área (DIGGLE, 2003). A análise foi feita graficamente para facilitar a visualização dos desvios em relação à hipótese nula, através de um gráfico cuja abscissa representa a escala e a ordenada a função transformada $L(r)$ da função $\mathrm{K}$.

\subsection{Produtividade de forragem}

A forragem produzida foi coletada sob a copa das árvores de pequi em áreas de pasto a pleno sol sem interferência de algum tipo de sombreamento, previamente ao pastejo do gado, ao final das estações de chuva e seca, períodos distintos consolidados para produção de pasto (PORTO et al., 2009). A primeira coleta (final da estação chuvoso) ocorreu no mês de abril e a segunda (final da estação seca) no mês de outubro de 2014. Para maior representatividade amostral da produção de matéria seca, visto a variabilidade de formatos de copa que o pequi apresentou no sistema silvipastoril, foram escolhidas por sorteio duas árvores por classe de AC, obtendo-se 10 pontos de coleta sob copa e 10 pontos em pleno sol.

Considerou-se como forragem todo material vegetal que compõem o pasto contido dentro do quadrado de $0,64 \mathrm{~m}^{2}$, usado para amostragem, e cortado a $10 \mathrm{~cm}$ do solo. A leguminosa estilosante, por mínima presença na quantificação da forragem na condição de sombreamento e pleno sol, foi retirada do estudo. As amostras foram fracionadas em lâmina foliar (LF), bainha + colmo (BC), e material senescente (MS) (área seca $>50 \%$ ), organizadas em sacos de papel, secas em estufa de circulação de ar forçada por 72 horas e pesadas em balança de precisão para obtenção do rendimento de matéria seca (RMS).

Os dados foram submetidos ao teste $\mathrm{F}$ com significância de 10\% (p-valor 0,1). Posteriormente, as médias foram submetidas ao Teste $\mathrm{t}$ para dados dependentes e dados independentes, com significância de $10 \%$.

\section{RESULTADOS}

$\mathrm{Na}$ área de estudo foram identificadas 139 árvores de pequi, não encontrando na população indivíduos com menos de $0,19 \mathrm{~m}$ e com máximo de $0,94 \mathrm{~m}$ de DAS, sendo a média de $0,51 \mathrm{~m}$ (Tabela 1). As medidas obtidas se diferem das observadas por Santana; Naves (2003) e Bruzinga et al. (2013) que encontraram nos respectivos levantamentos, diâmetro médio não maior que $0,15 \mathrm{~m}$. A média da $\mathrm{AC}$ dos pequizeiros foi de $148,73 \mathrm{~m}^{2}$ com indivíduos acima de $500 \mathrm{~m}^{2}$ (Tabela 2), valores esses mais altos do que os encontrados por Bruziguessi (2016) em propriedades rurais na região de cerrado, o qual constatou áreas de copa de pequizeiros com média de $110 \mathrm{~m}^{2}$.

Tabela 1. Amplitudes de altura total (Ht), altura de base da copa $(\mathrm{Hbc})$, diâmetro à altura do solo (DAS), diâmetro de copa (DC) e área de copa (AC) de indivíduos adultos de pequi associados a pastagem. Table 1. Amplitude of total height $(\mathrm{Ht})$, crown height $(\mathrm{Hbc})$, diameter of soil height (DAS), crown diameter (DC) and crown area (CA) of pequi adult individuals associated with pasture.

\begin{tabular}{ccccc}
\hline Variáveis & Máx. & Min. & Média & S \\
\hline $\mathrm{Ht}(\mathrm{m})$ & 17,00 & 4,00 & 10,22 & $( \pm 2,65)$ \\
$\mathrm{Hbc}$ & 5,20 & 0,80 & 2,26 & $( \pm, 074)$ \\
$\mathrm{DAS}(\mathrm{m})$ & 0,94 & 0,19 & 0,51 & $( \pm 0,16)$ \\
$\mathrm{DC}(\mathrm{m})$ & 26,00 & 1,00 & 13,25 & $( \pm 3,72)$ \\
$\mathrm{AC}\left(\mathrm{m}^{2}\right)$ & 530,93 & 0,79 & 148,73 & $( \pm 78,61)$ \\
\hline Desvio padrão: $\mathrm{S}$ & & & &
\end{tabular}

Tabela 2. Número de indivíduos adultos de pequi (NI) associados com pastagem. Classificação em função da área de copa (AC).

Table 2. Number of adult pequi (NI) individuals associated with pasture. Classification according to cup area (AC).

\begin{tabular}{ccccccc}
\hline \multicolumn{2}{c}{$\mathrm{AC}\left(\mathrm{m}^{2}\right)$} & $\mathrm{NI}$ & Mínimo & Máximo & Média & $\mathrm{S}$ \\
\hline 1 & 0,00 & 4,00 & 0,79 & 29,71 & 13,82 & $( \pm 12,69)$ \\
2 & 70,10 & 42,00 & 31,17 & 108,43 & 77,50 & $( \pm 23,08)$ \\
3 & 148,73 & 57,00 & 110,30 & 186,26 & 146,98 & $( \pm 22,77)$ \\
4 & 227,30 & 25,00 & 189,91 & 264,46 & 216,38 & $( \pm 22,90)$ \\
5 & 306,00 & 11,00 & 279,07 & 530,93 & 325,12 & $( \pm 72,33)$ \\
\hline \multicolumn{2}{c}{ Total } & 139,00 & 0,79 & 530,93 & 148,73 & $( \pm 78,61)$ \\
\hline
\end{tabular}

Desvio padrão: $\mathrm{S}$

As árvores de pequi tiveram média de 10,22 m de altura e quando distribuídas em classes de altura de um metro (Figura 2 A), notou-se a concentração de indivíduos nas classes 8-9 m; 9-10 m; 10-11 m, diferente de valores observados na literatura (SANTANA; NAVES, 2003; BRUZINGA et al., 2013; BRUZIGUESSI, 2016). A Hbc apresentou em média 2,26 m e notou-se que a maior parte das árvores têm mais de $2 \mathrm{~m}$ de altura base de copa (Figura 2 B).

Observou-se diferença significativa das frações LF, BC e total, na qual a condição de sombreamento das árvores de pequi teve maior RMS de forragem que a pleno sol, ao final da estação seca e chuvosa $(\mathrm{p}<0,1)$ (Tabela 3$)$.

A formação de adensamentos de indivíduos em áreas de plena exposição ao sol é observada de acordo com distribuição das árvores e em relação das classes de AC (Figura 3), fato que demonstra o potencial do pequi como componente arbóreo para o sistema silvipastoril, por haver a compatibilidade da 
espécie com o local e restrição parcial da luminosidade sob a copa, o que possibilita a associação com forrageiras. A partir dos resultados da função K de Ripley (Figura 4), observou-se que o intervalo da curva do valor $\mathrm{K}$ para a distribuição do pequi observado e as curvas do envelope de confiança têm o mesmo ponto inicial onde $\mathrm{L}(\mathrm{r})=0$ com valor de escala gerado de $150 \mathrm{~m}$.

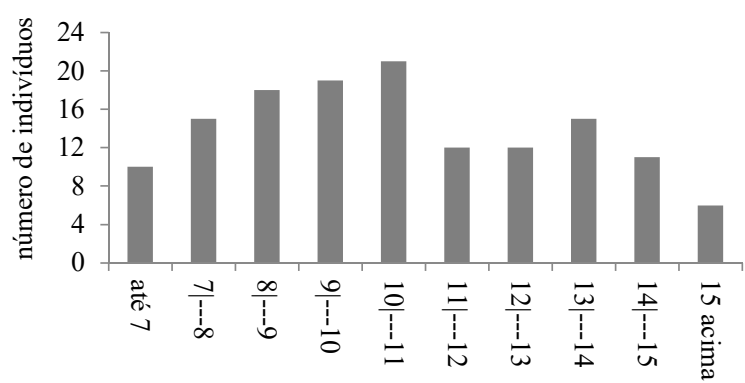

A $\mathrm{Ht}(\mathrm{m})$

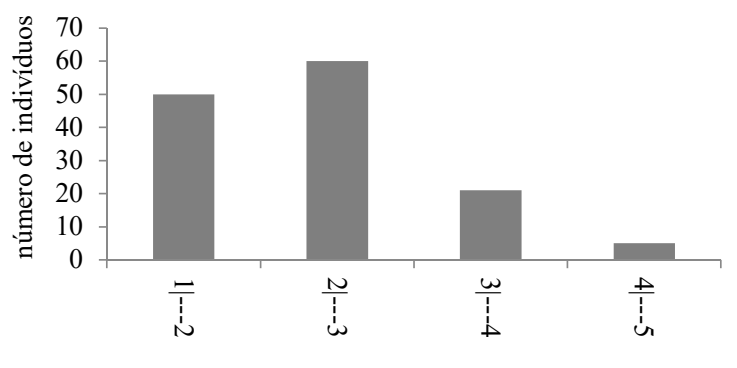

$\mathrm{Hbc}(\mathrm{m})$

Figura 2. Distribuição de indivíduos de pequi em altura total $(\mathrm{Ht}) \mathrm{e}$ altura de base da copa (Hbc) em área de pastagem. $\mathrm{Ht}(\mathrm{A})$; Hbc (B). Figure 2. Distribution of pequi individuals at total height $(\mathrm{Ht})$ and canopy base height ( $\mathrm{Hbc}$ ) in pasture area. $\mathrm{Ht}(\mathrm{A})$; $\mathrm{Hbc}(\mathrm{B})$.

Tabela 3. Rendimento de matéria seca de forragem em sistema silvipastoril com pequi no final da estação de seca e no final da estação chuvosa.

Table 3. Dry matter yield of forage in silvopastoral system with pequi at the end of the dry season and at the end of the rainy season.

\begin{tabular}{|c|c|c|c|c|c|}
\hline \multirow{2}{*}{\multicolumn{2}{|c|}{$\begin{array}{c}\text { Rendimento de } \\
\text { matéria seca }\left(\mathrm{kg} \mathrm{ha}^{-1}\right)\end{array}$}} & \multicolumn{2}{|c|}{ Estimativa } & \multirow{2}{*}{$t$} & \multirow{2}{*}{$\operatorname{Pr}(>|t|$} \\
\hline & & Sombra & Pleno sol & & \\
\hline \multirow{4}{*}{ ల్ల } & LF & $2421,7 \mathrm{a}$ & $2087,8 \mathrm{~b}$ & 1,7320 & 0,0586 \\
\hline & $\mathrm{BC}$ & $3255,5 \mathrm{a}$ & $2527,4 \mathrm{~b}$ & 2,2164 & 0,0269 \\
\hline & MS & $1664,3 \mathrm{a}$ & $1827,0 \mathrm{a}$ & 1,0117 & 0,1690 \\
\hline & Total & $7341,5 \mathrm{a}$ & $6442,2 \mathrm{~b}$ & 1,6629 & 0,0654 \\
\hline & LF & $4812,8 \mathrm{a}$ & $3982,3 \mathrm{~b}$ & 2,6085 & 0,0142 \\
\hline & $\mathrm{BC}$ & $3620,1 \mathrm{a}$ & $2469,9 \mathrm{~b}$ & 5,2669 & 0,0003 \\
\hline & MS & $2372,8 \mathrm{~b}$ & $2899,0 \mathrm{a}$ & 3,2764 & 0,0048 \\
\hline & Total & $10820,7 \mathrm{a}$ & $9351,2 \mathrm{~b}$ & 2,7981 & 0,0104 \\
\hline
\end{tabular}

Amostras fracionadas em lâmina foliar (LF), bainha + colmo (BC), e material senescente (MS). Letras iguais na linha não diferem entre si pelo teste $t, p<$ 0,1 .

\section{DISCUSSÃO}

O bioma cerrado tem como vegetação estratos contínuos de espécies herbáceas e lenhosas, com altura entre dois e oito metros (RIBEIRO; WALTER, 2008). Fatores intrínsecos como escala ecológica e/ou evolutiva e/ou extrínsecos por se tratarem de indivíduos remanescentes, podem justificar a presença de indivíduos com mais de 10 metros de altura como observado neste estudo.

A altura e a área de copa são variáveis importantes para a consolidação do sistema silvipastoril, visto as mudanças ocorridas no pasto e no ambiente de pastejo (GARCEZ-NETO et al., 2010; KARVATTE JUNIOR et al., 2016). No caso da associação de espécies arbóreas nativas com pasto, há poucos trabalhos sobre este assunto e que estudam detalhadamente a altura e a área de copa das árvores.

A proporção de árvores de pequi dispersas sobre a área total do sistema silvipastoril é de 3,5 árvores de pequi ha- ${ }^{-1}$, e quando se soma os valores totais de $\mathrm{AC}$, estes representam 5\% da área com sombreamento sobre a forragem. Não há ainda um número ideal de árvores que pode estar associado ao sombreamento de pastos, principalmente para espécies de cerrado, e na maioria dos casos, por falta de conhecimento sobre a importância da biodiversidade, esse número é reduzido ou quase nulo. Áreas de pastejo com pouco ou nenhum sombreamento tendem a concentrar os animais no momento de ruminação em locais específicos como porteiras, aguadas e cochos por longos períodos, o que acarreta em concentração de fezes e urina nesses locais, culminando em possíveis doenças, além da compactação do solo (FERREIRA et al., 2011, ANDRADE et al., 2012).

O total de indivíduos e as classes de AC $2\left(70,1 \mathrm{~m}^{2}\right), 3$ $\left(148,7 \mathrm{~m}^{2}\right)$ e $4\left(227,30 \mathrm{~m}^{2}\right)$, tiveram o valor $K$ acima do envelope de confiança. O número de vizinhos próximos a uma árvore aleatória de pequi está acima do previsto para a CAE e assim, os indivíduos adultos de pequi analisados e as classes estudadas apresentam padrão de distribuição espacial agregada, como constatado por Bruzinga et al. (2013), pois o número de indivíduos observado na proximidade de um indivíduo qualquer foi maior do que o esperado para um padrão aleatório.

A classe $1\left(0,00 \mathrm{~m}^{2}\right)$ apresentou agregação até $100 \mathrm{~m}$ e tendência a aleatoriedade desta distância até $150 \mathrm{~m}$, e na classe $5\left(306 \mathrm{~m}^{2}\right)$ ocorreu o inverso, demonstrou distribuição aleatória até $60 \mathrm{~m}$ e agregação deste ponto em diante, devido ao estádio de desenvolvimento dentro da população. O pequi apresenta padrão agregado de distribuição das árvores de maior AC (mãe) em relação as árvores de menor AC (filhos), e a aleatoriedade ocorre apenas nas árvores com maior AC.

Este distanciamento entre indivíduos, assim como foi encontrado neste estudo, forma o denominado mosaico vegetacional (SILVA et al., 2012), o que é interessante para consolidar o sistema silvipastoril, principalmente no diz respeito ao bem-estar animal (KARVATTE JUNIOR et al., 2016). A formação de bosquetes sobre o pasto cria condição microambiental diferente de pastos homogêneos (ABRAHAM et al., 2014), pois há considerável redução da insolação e temperatura, fatores que interagem com o hábito ingestivo e desempenho animal (TITTO et al., 2011; SILVA et al., 2013).

O desenvolvimento da forragem depende da tolerância a restrição de luminosidade, além da precipitação e a umidade relativa do local onde é estabelecida. A queda de produção de forragem ocorreu como esperado no período de seca. No entanto, em locais com sombreamento do pequi, reduziu-se este efeito sobre o pasto, acumulando maior quantidade de matéria seca em relação à condição de pleno sol. Tal resultado é observado também para o período chuvosos, e isso demonstra que além da tolerância ao sombreamento, as gramíneas foram beneficiadas pela associação com as árvores de pequi. Oliveira et al. (2005) e Calil et al. (2016) não constaram redução do RMS de Brachiaria decumbens em associação com pequi. Todavia, os autores destacaram a importância da espécie no sistema silvipastoril ao incremento nutricional no pasto sob a copa e seu entorno devido à deposição de serapilheira. 

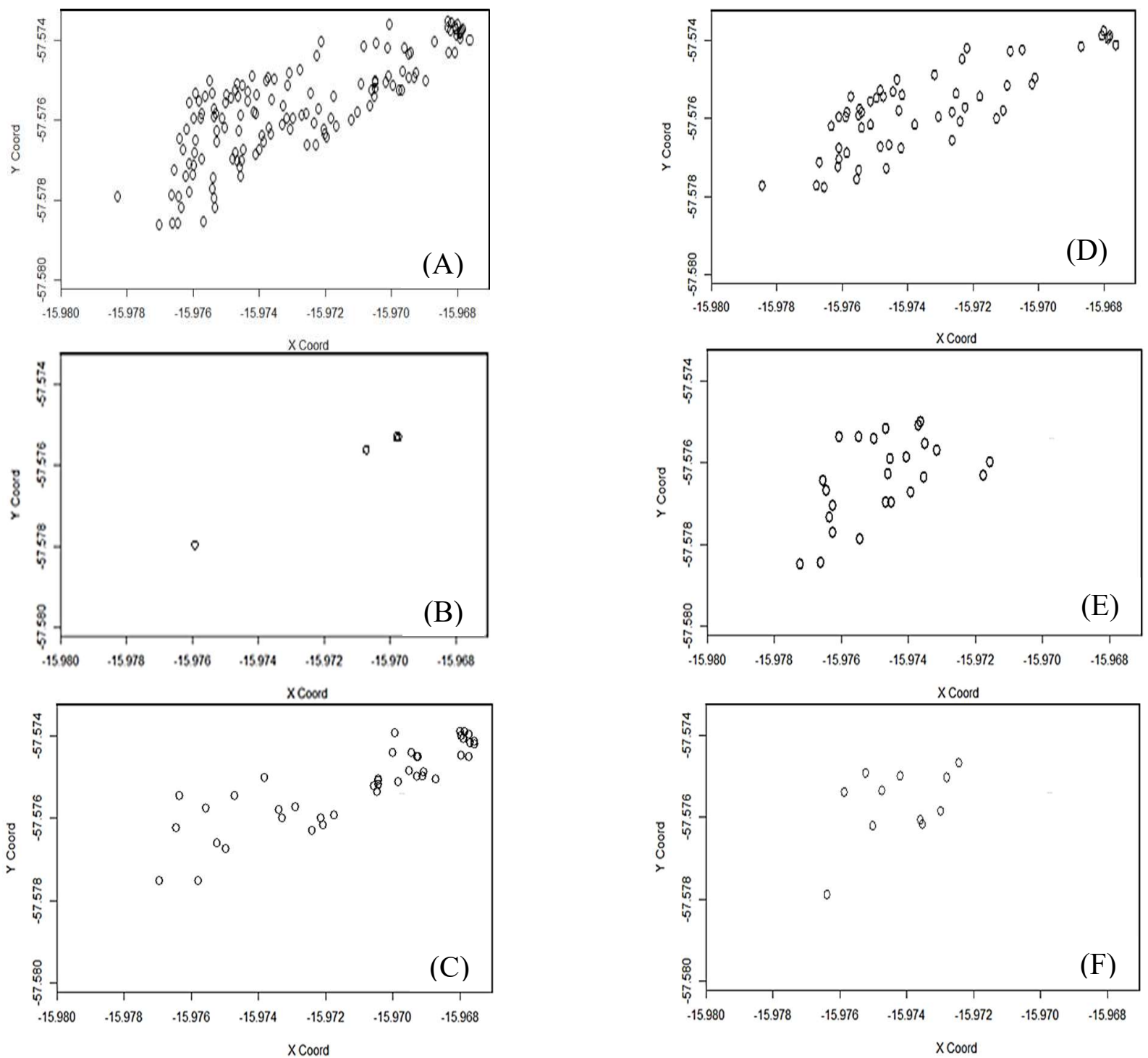

Figura 3. Distribuição espacial dos indivíduos de pequi associados a forrageiras. (A) Total de indivíduos; (B) classe $1-0,00 \mathrm{~m}^{2}$ de AC; (C) classe 2 - 70,1 $\mathrm{m}^{2}$ de AC; (D) classe 3 - 148,7 $\mathrm{m}^{2}$ de AC; (E) classe $4-227,30 \mathrm{~m}^{2}$ de AC; (F) classe 5 - $306 \mathrm{~m}^{2}$ de AC.

Figure 3. Spatial distribution of pequi individuals associated with forages. (A) Total individuals; (B) class $1-0.00 \mathrm{~m}^{2} \mathrm{AC}$; (C) class 2 - 70.1 $\mathrm{m}^{2}$ of AC; (D) class $3-148.7 \mathrm{~m}^{2} \mathrm{AC}$; (E) class $4-227.30 \mathrm{~m}^{2} \mathrm{AC}$; (F) class $5-306 \mathrm{~m}^{2} \mathrm{AC}$.

A fração MS diferenciou-se estatisticamente apenas ao final da estação chuvosa, com redução de $18,15 \%$ de RMS $\left(500,26 \mathrm{~kg} \mathrm{ha}^{-1}\right)$ da condição sombreada em relação ao pleno sol. A senescência é um processo metabólico determinado pela genética da planta e em função da ação da temperatura ambiente sobre o meristema apical (TAIZ; ZEIGER, 2013). Assim, na condição sombreada, as características fisiológicas da forragem são alteradas ao ponto de prolongar o ciclo vegetativo da forragem.

\section{CONCLUSÕES}

A distribuição espacial da população de pequi remanescente na área onde foi estabelecido o sistema silvipastoril é do tipo agregado.

Os bosquetes caracterizados em função da área de copa geram benefícios para o sistema silvipastoril.

O sombreamento promovido pela copa das árvores de pequi favoreceu a produção do pasto nos períodos de seca e chuva, com redução do material senescente.

\section{REFERÊNCIAS}

ABRAHAM, E. M.; KYRIAZOPOULOS, A. P.; PARISSI, Z. M.; KOSTOPOULOU, P.; KARATASSIOU, M.; ANJALANIDOU, K.; KATSOUTA, C. Growth, dry matter production, phenotypic plasticity, and nutritive value of three natural populations of Dactylis glomerata L. under various shading treatments. Agroforestry Systems, Dordrecht, v. 88, n. 2, p. 287-299, 2014. DOI: https://dx.doi.org/10.1007/s10457-014-9682-9

ABREU, S. A. H.; ARRUDA, E. M.; BARROS, L. R.; ALMEIDA, R. F.; MARANHÃO, D. D. C.; SILVA, V. L.; SILVA-NETO, C. M.; FLORES, R. A.; CALIL, F. N.; COLLIER, L. S. Chemical attributes of the soil in agroforestry systems subjected to organic fertilizations. African Journal Agricultural Research, Nairobi, v. 11, n. 27 p. 2378-2388, 2016. DOI: https://dx.doi.org/10.5897/AJAR2016.11182 

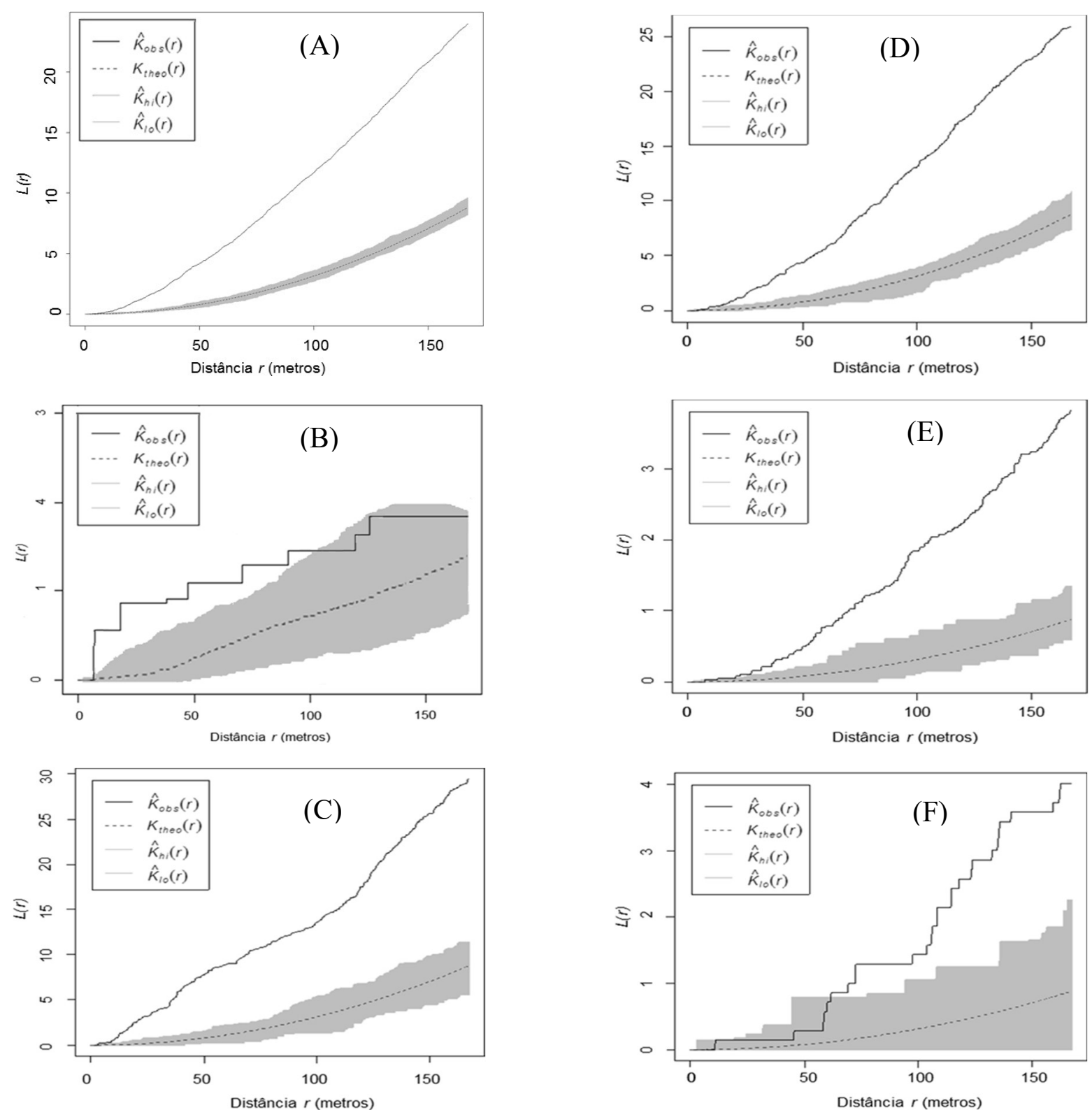

Figura 4.Função $K$ de Ripley aplicada em indivíduos adultos de pequi em sistema silvipastoril. (A) Total de indivíduos; (B) classe $1-0,00 \mathrm{~m}^{2}$ de AC; (C) classe 2 - 70,1 $\mathrm{m}^{2}$ de AC; (D) classe 3 - 148,7 $\mathrm{m}^{2}$ de AC; (E) classe 4 - 227,30 $\mathrm{m}^{2}$ de AC; (F) classe 5 - $306 \mathrm{~m}^{2}$ de AC. Na legenda: $\hat{K}_{\text {obs }}(\mathrm{r})$ (linha contínua); Valor da função $K$ observada; Ktheo(r) (linha tracejada): Valor teórico para a função $K$; $\hat{K}_{\text {hi(r) }}$ e $\hat{K}_{\text {lo }}(\mathrm{r})$ (linhas sombreadas): Respectivamente, limite superior e limite inferior do envelope de confiança.

Figure 4. Ripley'sK function applied in pequi adult individuals in a silvopastoral system. (A) Total individuals; (B) class $1-0.00 \mathrm{~m}^{2} \mathrm{AC}$; (C) class $2-70.1 \mathrm{~m}^{2}$ of AC; (D) class $3-148.7 \mathrm{~m}^{2} \mathrm{AC}$; (E) class $4-227.30 \mathrm{~m}^{2} \mathrm{AC}$; (F) class $5-306 \mathrm{~m}^{2} \mathrm{AC}$. In the legend: $\hat{K}_{\text {obs }}(\mathrm{r})$ (continuous line); Value of observed $K$ function; $K_{\text {theo }}(\mathrm{r})$ (dashed line): Theoretical value for the function $K ; \hat{K}_{\mathrm{hi}}(\mathrm{r})$ e $\hat{K}_{\mathrm{lo}}(\mathrm{r})$ (shaded lines): Respectively, upper limit and lower bound of the trust envelope.

AFONSO, S. R.; ANGELO, H. Mercado dos produtos florestais não madeireiros do cerrado brasileiro. Ciência Florestal, Santa Maria, v. 19, n. 3, p. 315-326, 2009. DOI: http://dx.doi.org/10.5902/19805098887

ALMG - ASSEMBLEIA LEGISLATIVA DE MINAS GERAIS. Disponível em: <https://www.almg.gov.br/co nsulte/legislacao/completa/completa.html?ano=1992\&nu $\mathrm{m}=10883 \&$ tipo $=$ LEI\&aba $=$ js_textoOriginal $>$. Acesso em: 3 mai 2018.

ANDRADE, C. M. S.; SALMAN, A. K. D.; OLIVEIRA, T. K. Guia arbopasto: manual de identificação e seleção de espécies arbóreas para sistemas silvipastoris. Brasília: Embrapa, 2012. 345 p

ASSUNÇÃO, R. M.; LOPES, D. Medida de correlação entre padrões pontuais de origem-destino. In: VIII Brazilian Symposium on GeoInformatics, Campos do Jordão, Brazil, INPE, p. 315-320. 2006. Anais... Campos do Jordão: GeoInfo, 2006. Disponível em: <http://mtcm16c.sid.inpe.br/col/dpi.inpe.br/geoinfo@80/2006/11.28. 12.13/doc/315-320.pdf $>$. Acesso em: 22 de outubro de 2018.

BADDELEY, A. Analysing spatial point patterns in $\mathbf{R}$. Camberra: CSIRO, 2010.232 p. (Technical report). 
BATISTA, J. L. F.; MAGUIRE, D. A. Modeling the spatial structure of tropical forests. Forest Ecology and Management, Amsterdam, v. 110, n. 1-3, p. 293-314, 1998. DOI: https://doi.org/10.1016/S03781127(98)00296-5

BRUZIGUESSI, E. P. Árvores nativas do cerrado na pastagem: Por quê? Como? Quais? 2015. 163f. Tese (Doutorado em Ciências Florestais) - Universidade de Brasília, Brasília, 2016.

BRUZINGA, J. S.; OLIVEIRA, M. L. R.; MACHADO, E. L. M.; LEITE, H. G.; PEREIRA, I. M.; NOGUEIRA, G. S. Distribuição espacial de indivíduos adultos de pequi. Scientia Forestalis, Piracicaba, v. 41, n. 98, p. 249-256, 2013.

CALIL, F. N.; LIMA, N. L.; SILVA, R. T.; MORAIS, M. D. A.; BARBOSA, P. V. G.; LIMA, P. A. F.; BRANDÃO, D. C.; SIlvA-NETO, C. M.; CARVALHO, H. C. S.; NASCIMENTO, A. R. Biomass and nutrition stock of grassland and accumulated litter in a silvopastoral system with Cerrado species. African Journal of Agricultural Reseach, Nairobi, v. 11, n. 38, p. 3701-3709, 2016. DOI: https://dx.doi.org/10.5897/AJAR2016.11369

CÂMARA, G.; CARVALHO, M. S. Análise Espacial de Eventos. In: DRUCK, S.; CARVALHO, M. S.; CÂMARA, G.; MONTEIRO, A. M. V. (Ed.). Análise espacial de dados geográficos. Planaltina: EMBRAPA, 2004. p. 55-75.

DIGGLE, P. J. Statistical analysis of spatial point patterns. London: Academic Press, 2003. 272 p.

EMBRAPA - EMPRESA BRASILEIRA DE PESQUISA AGROPECUÁRIA. Sistema brasileiro de classificação de solos. 2 ed. Rio de Janeiro: Embrapa - Centro Nacional de Pesquisa de Solos, 2006, 306p.

FERREIRA, L. C. B.; MACHADO FILHO, L. C. P.; HOETZEL, M. J.; LABARRÈRE, J. G. O efeito de diferentes ofertas de sombra na dispersão das fezes dos bovinos nas pastagens. Revista Brasileira de Agroecologia, Cruz Alta, v. 6, n. 1, 2011.

GARCEZ-NETO, A. F.; GARCIA, R.; MOOT, D. J.; GOBBI, K. F. Aclimatação morfológica de forrageiras temperadas a padrões e níveis de sombreamento. Revista Brasileira de Zootecnia, Pelotina, v. 39, n. 1, 2010.

HARVEY, C. A.; VILLANUEV, C.; ESQUIVEL, H.; GÓMEZ, R.; LOPEZ, M. I. M.; MARTINEZ, J.; MUÑOZ, D.; RESTREPO, C.; SAÉNZE, J. C.; VILLACÍS, J.; SINCLAIRFG, F. Conservation value of dispersed tree cover threatened by pasture management. Forest Ecology and Management, Amsterdam, v. 261, p. 1664-1674, n. 10, $2011 . \quad$ DOI: https://doi.org/10.1016/j.foreco.2010.11.004

IBAMA - INSTITUTO BRASILEIRO DO MEIO AMBIENTE E DOS RECURSOS NATURAIS RENOVÁVEIS. Biblioteca digital do meio ambiente. Disponível em: <http://www.ibama.gov.br/sophia/cnia/ legislação/IBDF/PT0054-050387.pdf> . Acesso: 3 de maio de 2018.

KARVATTE JUNIOR, N.; KLOSOWSKI, E. S.; ALMEIDA, R. G.; MESQUITA, E. E.; OLIVEIRA, C. C.; ALVES, F. V. Shading effect on microclimate and termal comfort indexes in integrated crop-livestock-forest systems in the Brazilian Midwest. International Journal of Biometeorology, Lisse, v. 60, n. 12, p. 1-9, 2016. DOI: https://dx.doi.org/10.1007/s00484-016-1180-5
KICHEL, A. N.; COSTA, J. A. A.; ALMEIDA, R. G.; PAULINO, V. T. Sistema de integração lavoura-pecuáriafloresta (ILPF) - experiências no Brasil. Boletim de Indústria Animal, Nova Odessa, v. 71, n. 1, p. 94-105, 2014.

MEIRA, M. R.; CABACINHA, C. D.; GAMA, A. T.; MARTINS, E. R.; FIGUEIREDO, L. S. Caracterização estrutural do barbatimão (Stryphnodendron adstringens (Mart.) Coville) no cerrado do norte de Minas Gerais. Ciência Florestal, Santa Maria, v. 26, n. 2, p. 627-638, 2016. DOI: http://dx.doi.org/10.5902/1980509822762

OLIVEIRA, M. E.; LEITE, L. L.; CASTRO, L. H. R. Efeito de duas espécies nativas de árvores sobre as propriedades do solo e forragem de Brachiaria decumbens Stapf.. Pasturas Tropicales, Cali, v. 27, n. 1, p. 51-56, 2005.

PORTO, M. O.; PAULINO, M. F.; LEÃO, M. I.; COUTO, V. R. M. Fontes suplementares de proteína para novilhos mestiços em recria em pastagens de capim-brachiaria no período das águas: desempenho produtivo e econômico. Revista Brasileira de Zootecnia, Viçosa, v. 38, n. 8, p. 1553-1560, 2009. DOI: http://dx.doi.org/10.1590/S151635982009000800020

RIBEIRO, J. F.; WALTER, B. M. T. Fitofisionomias do bioma Cerrado. In: SANO, S. M.; ALMEIDA, S. P. de; RIBEIRO, J. F. (Ed.). Cerrado: ecologia e flora. Planaltina: EMBRAPA-CPAC, 2008. p.151-212.

RIPLEY, B. D. Spatial statistics. New York: Wiley-IEEE, 1981. 252p.

SANTANA, J. das G. E.; NAVES, R. V. Caracterização de ambientes de cerrado com alta densidade de pequizeiros (Caryocar brasiliense Camb.) na região sudeste do estado de Goiás. Pesquisa Agropecuária Tropical, Goiânia, v. 33, p. 1-10, 2003

SCOLFORO, J. R. S.; MELLO, J. M. Inventário Florestal. Lavras: UFLA-FAEPE, 2006. 561p.

SILVA, K. E.; MARTINS, S. V.; SANTOS, N. T.; RIBEIRO, C. A. A. S. Padrões espaciais de espécies arbóreas tropicais. In: MARTINS, S. V. (Ed.). Ecologia de florestas tropicais do Brasil. 2. ed. rev. E aum. Viçosa: UFV, 2012. $371 \mathrm{p}$.

SILVA, L. L. G. G. da; RESENDE, A. S. de; DIAS, P. F.; SOUTO, S. M.; AZEVEDO, B. C. de; VIEIRA, M. de S.; COLOMBARI, A. A.; TORRES, A. Q. A.; MATTA, P. M. da; PERIN, T. B.; CAMPELLO, E. F. C.; FRANCO, A. A. Comportamento ingestivo diurno de novilhas mestiças em sistema silvipastoril em uma região tropical. Archivos Latinoamericanos de Producción Animal, Montevideo, v. 21, n. 1, p.15-22, 2013.

TITTO, C. G.; TITTO, E. A. L.; TITTO, R. M.; MOURÃO, G. B. Heat tolerance and the effects of shade on the behavior of Simmental bulls on pasture. Animal Science Journal, v.82, p.591-600, 2011. DOI: https://dx.doi.org/10.1111/j.1740-0929.2011.00872.x

TAIZ, L.; ZEIGER, E. Fisiologia vegetal. 5. ed. Porto Alegre, Artmed, 2013.918 p.

VALÉRIO, I. P.; CARVALHO, F. I. F. de; OLIVEIRA, A. C. de; SOUZA, V. Q.; BENIN, G.; SCHMIDT, D. A. M.; RIBEIRO, G.; NORNBERG, R.; LUCH, H. Combining ability of wheat genotypes in two models of diallel analysis. Crop Breeding and Applied Biotechnology, v. 9, n. 2, p. 100-107, 2009. DOI: https://dx.doi.org/10.12702/1984-7033.v09n02a01 